\section{Trps1-deficient transplanted skin gave rise to a substantial amount of hair: Trps1 is unnecessary for hair development}

\author{
Yingzhe Zhang, ${ }^{1}$ \\ Tomoyuki Nakamura, ${ }^{2}$ \\ Fukumi Furukawa, ${ }^{2}$ \\ Yasuteru Muragaki ${ }^{1}$
}

${ }^{1}$ Department of Pathology; ${ }^{2}$ Department of Dermatology, Wakayama Medical

University School of Medicine, Japan

\begin{abstract}
Trps1 is considered as an important gene involved in the interactions between the epithelial and mesenchymal cells during hair follicle morphogenesis. The number of hair follicles in Trps1 Knockout (KO) newborn mouse skin was significantly lower than that in wild-type (WT) newborn skin. To gain insight into the functional role of Trps1 in hair development, we transplanted Trps 1 KO newborn mouse skin on the backs of nude mice and examined hair growth at day 42 after transplantation. Surprisingly, transplanted skin from Trps1 KO newborn mice gave rise to a substantial amount of hair, although the hair was softer than that of WT mice. Histological examination revealed that the diameter of both hair follicles and hair shafts were significantly lower, whereas the density of hair follicles showed no significant difference between the Trps1 KO and WT mice. We introduce mouse hair follicles as a fascinating model to study the functions of Trps1 in mouse hair growth and pathology. This model suggests that the function of Trps1 is unnecessary for the development of normal hair follicles and hair shafts, although the loss of Trps 1 affects the diameters of hair follicles and hair shaft.
\end{abstract}

\section{Introduction}

It has been reported that heterozygous germ line mutations in tricho-rhino-phalangeal syndrome (Trps1) on chromosome $8 \mathrm{q} 23$ in humans result in autosomal dominant inheritance of tricho-rhino-phalangeal syndrome type I (TrpsI) and III (TrpsIII), characterized by sparse and slow growing scalp hair, as well as craniofacial and skeletal abnormalities. ${ }^{1,2}$ Trps1 Knockout (KO; it is a genetically modified mouse, Musmusculus, in which researchers have inactivated, or knocked out, an existing gene by disrupting it with an artificial piece of DNA) mice were reported to have fewer hair follicles, with craniofacial and skeletal defects that mirror the phenotypic characteristics of human patients. Patients with TRPS I have an orbicular nose, a long and even philtrum, a thin upper lip, sparse scalp hair that grows slowly, and protruding ears. 3,4

These findings proved that Trps1 was required for specific aspects of hair growth regulation. In addition to the apparent defects in facial soft tissues, male patients were particularly affected by hair loss, with many being nearly or completely bald soon after puberty. Some children with this disease have loose skin, although the skin becomes tighter over time. Individuals with TRPS I may experience excessive sweating, although the clinical description is often incomplete. . $^{5-8}$

Skin development is a complex dynamic process that includes formation of epidermis, a layered self-renewing epithelium, and several skin auxiliaries such as hair follicles (HF), hair nails and sweat glands. HF morphogenesis is driven by bidirectional ectodermal-mesenchymal interactions between epidermal keratinocytes and a specialized population of dermal fibroblasts, resulting in formation of the hair bulb, in which epithelial progenitor cells proliferate and differentiate into cell lineages to form hair shafts and their supporting layers in the inner root sheath. HF morphogenesis is governed by a well-balanced mutual effect among cell proliferation, differentiation, and apoptosis, all of which are controlled at several levels including signalling/transcription factor-mediated and epigenetic regulatory mechanisms. ${ }^{9,10}$

We previously demonstrated that transcription factor Trps1 played an important role in the morphogenesis of secondary HFs via an interaction with the BMP inhibitor Noggin. ${ }^{11}$ We found that development of secondary hair follicles in mutant Trps1 embryos was inhibited compared to their wide-type counterparts. Additional analysis revealed that Trps1 activated Wnt inhibitors and other transcription factors essential for follicle morphogenesis in mice. ${ }^{12}$ While this study demonstrated a requirement for Trps1 during early $\mathrm{HF}$ formation, it did not address the mechanisms underlying hair follicle degeneration in subsequent stages after birth. In addition, since Trps $1 \mathrm{KO}$ mice die within a few hours due to respiratory failure, we do not know whether or not hair grows with total loss of Trps1. In this study, to observe hair follicle growth after birth, we transplanted Trps1 KO newborn mouse skin to the back of nude mice.
Correspondence: Yasuteru Muragaki, Department of Pathology, Wakayama Medical University School of Medicine, 811-1 Kimiidera, Wakayama 641-0012, Japan. Tel.: 073.447.2300 - Fax: 073.447.2300.

E-mail:ymuragak@wakayama-med.ac.jp

Key words: Hair follicle; Trps1; Hair development; Hair shaft.

Contributions: YZ, TN, FF data collecting and analyzing; YZ, manuscript writing and references search.

Conflict of interest: the authors declare no potential conflict of interest.

Funding: this work was supported in part by a Grant-in-Aid for Scientific Research (15K08430) from the Ministry of Education, Science, Sports, and Culture of Japan (to Y.M.). It was also supported by CSC scholarship (NO. 201406220175).

Received for publication: 28 August 2018. Revision received: 18 December 2018.

Accepted for publication: 18 December 2018.

This work is licensed under a Creative Commons Attribution-NonCommercial 4.0 International License (CC BY-NC 4.0).

(C) Copyright Y. Zhang et al., 2019

Licensee PAGEPress, Italy

Dermatology Reports 2019; 11:7853

doi:10.4081/dr:2019.7853

\section{Materials and Methods}

\section{Trps1 Knockout mice and tissue preparation}

Trps1 KO mice were generated as previously described. ${ }^{13}$ The principles of animal care and use were followed as directed by the Committee of Wakayama University. We crossed male and female heterozygous mice to obtain wild-type and homozygous newborns. ${ }^{11}$ When newborn mice were harvested, genotyping was performed. Dorsal skin was carefully peeled off newborn mice and skin (6-mm in diameter) was taken with a tissue puncher and transplanted to the back of nude mice. 40 skin grafts from wild-type, heterozygous, and homozygous newborn, respectively, were transplanted to 20 nude mice (6 skin grafts per nude mouse). The skin graft was affixed to the recipient skin by adding Aron Alfa (KONISHI, Japan) at the margin.

\section{Preparation of histological sections and immunohistochemistry}

At day 42 after transplantation, transplanted skin tissue was obtained and fixed with $4 \%$ paraformaldehyde overnight. The 
next day, it was embedded in paraffin blocks. The skin was sectioned longitudinally into 5 - $\mu \mathrm{m}$ sections for HE staining or immunostaining.

\section{Statistical analysis}

The data were analyzed by Student's ttest with a Student-Newman-Keuls test (SPSS, 13.0). $\mathrm{P}<0.05$ was considered to be statistically significant.

\section{Results}

Abnormalities in appearance and morphogenesis of hair follicles in newborn Trps1 knockout mice

As we reported in a previous paper, newborn Trps1 KO mice showed a decreased size of the maxillary region compared with that of wild-type (WT; it refers to the phenotype of the typical form of a species as it occurs in nature) mice. ${ }^{11}$ Hair follicles in Trps1 KO mice were present at postnatal day1, but were reduced in number, irregularly spaced and smaller than those of WT mice (Figure 1).

Trps1 knockout mice showed distinct appearance differences at day 42 after transplantation

To investigate whether or not hair follicles develop and give rise to hair, and if Trps1 KO mice could survive, we transplanted newborn mouse skin to nude mice. Surprisingly, at day 42 after transplantation, transplanted skin from Trps $1 \mathrm{KO}$ newborn mice produced hairs with the same length and density as those of WT mice although they were softer than those of WT mice (Figure 2).

\section{Comparison between Trps1}

knockout and wild-type mice at day 42 after transplantation

Histological examination revealed that hair follicles and hairs were thinner in the transplanted skin from Trps1 KO newborn mice than those from WT mice (Figure 3A). Diameters of both hair follicles and hair shafts were significantly smaller in transplanted skin from Trps1 KO mice than those from WT mice (Figure 3B-D).

\section{Discussion and Conclusions}

In this study, we investigated whether hair could develop in a condition of Trps1deficient hair follicles. We expected that few hairs would emerge from transplanted
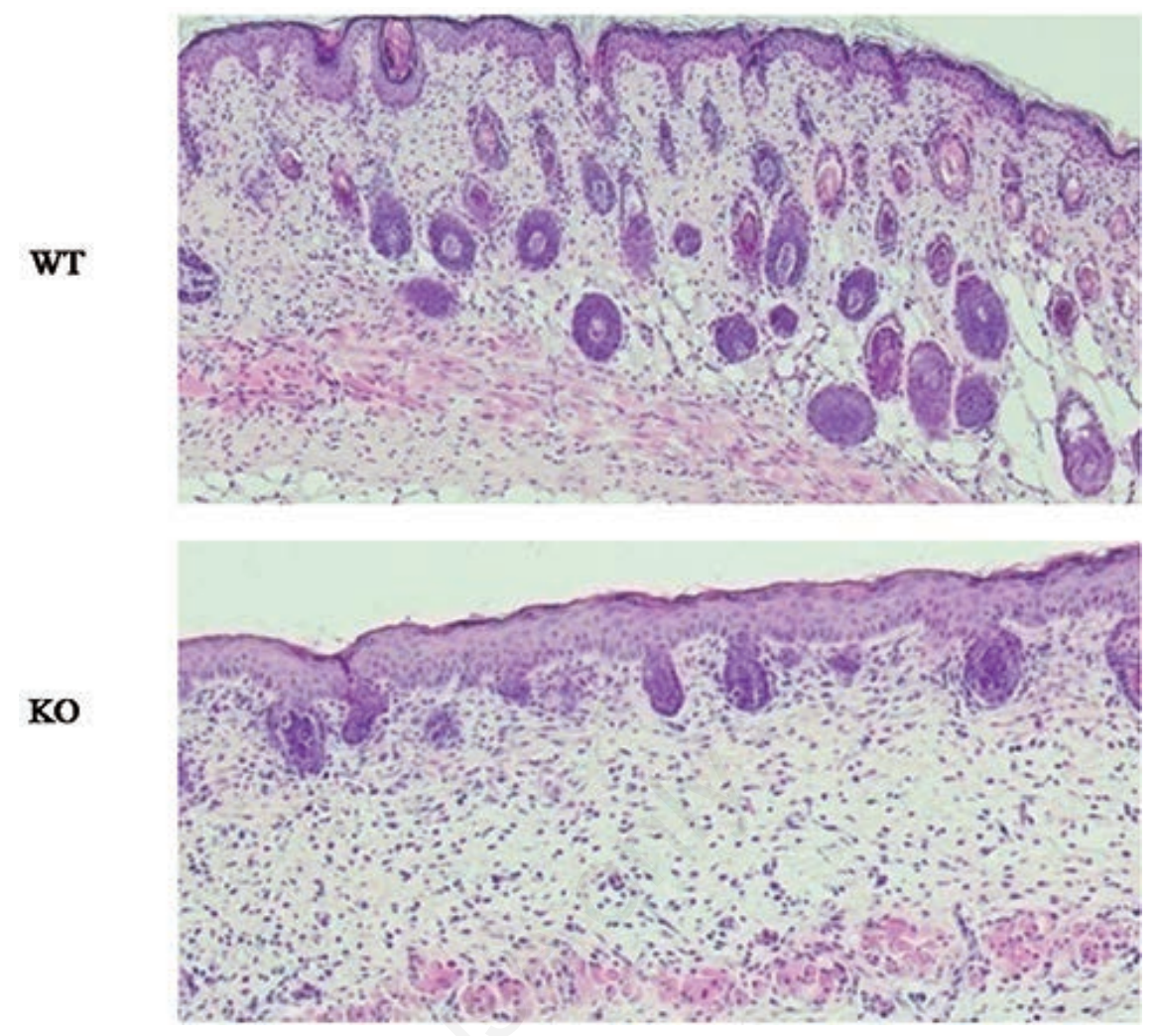

Figure 1. H\&E staining showing wild-type (WT) and Trps1 Knockout (KO) mice display differences during hair growth. 20x magnification. Histology of newborn dorsal skin got from WT and KO newborn mice. Note that Trps1 KO mice lack a number of hair follicles in newborn mice.

A
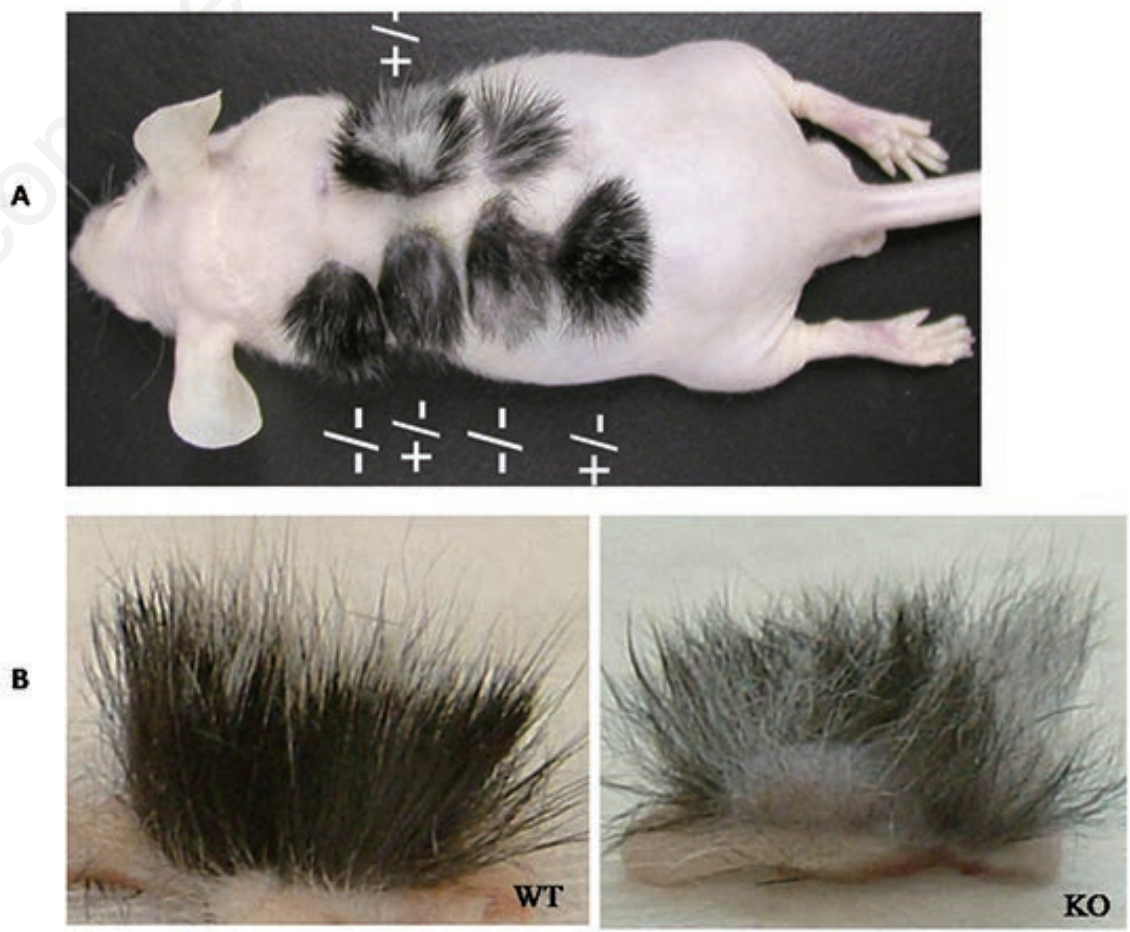

Figure 2. Appearance comparison of WT and Trps1 KO mice after 42 days of transplantation. (A) - I- shows the KO mouse transplanted skin, +/- shows the WT mouse transplanted skin; (B) Appearance of WT (left) and KO (right) mouse skin sample at 42 days after transplantation by naked-eye observing. Trps $1 \mathrm{KO}$ mouse sample showed a thinner and softer hair. 
skin from Trps1 KO newborn mice, because hair follicles in Trps $1 \mathrm{KO}$ newborn mice showed decreased number and diameter compared to those of WT mice. At day 42 after transplant, however, transplanted skin from Trps1 KO newborn mice produced soft, otherwise comparable amounts of hair as that of WT mice. Histological examination revealed smaller diameter of a hair follicle in Trps1 KO mice than that of WT mice.

In our previous papers, we provided evidence that Trps 1 expression was essential for normal hair follicle growth using Trps1 KO mice. ${ }^{11}$ Trps1 KO mice die shortly after birth due to respiratory insufficiency. These developmental abnormalities may be related to disrupted Wnt signalling. ${ }^{14}$ How disrupted Wnt signalling caused this abnormality led us to investigate the function of Trpslin hair follicle growth. The extensive phenotypic outcomes in Trps1 KO mice suggested that the structure of Trps1 protein might be required in the embryonic development of complex organ
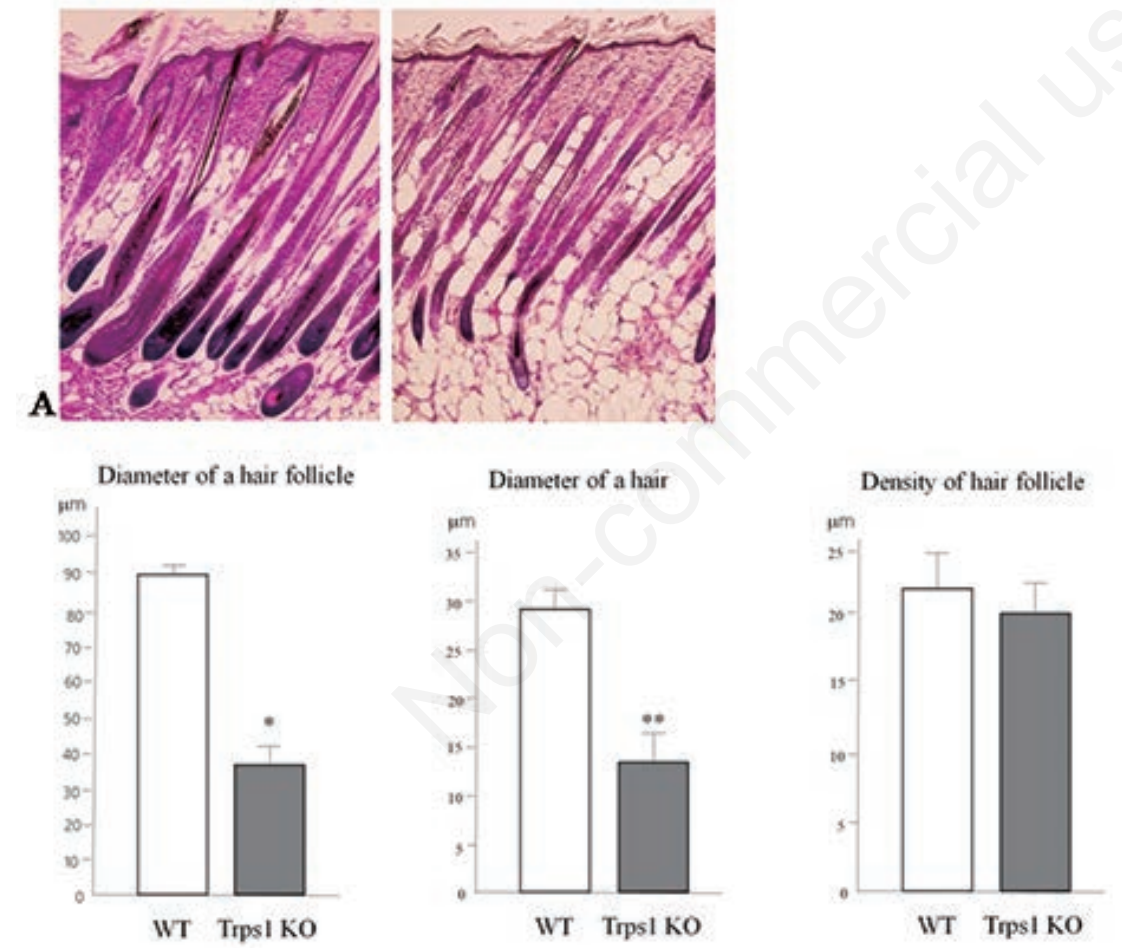

B $\quad * 0.001$

C $\quad * p=0.005$

D

Figure 3. Hair follicles are attenuated in Trps1 KO mice skin compared with those of WT mice at day 42 after transplantation. (A) H\&E staining of P42 WT and KO dorsal skin after transplant. Compared with WT (left), KO (right) displayed thinner hair shaft and shrunken hair follicles. 20x magnification. (B) Comparative analysis of diameter of hair follicles ( $n=3$ per group, error bars represent $\left.S D ;{ }^{*} \mathbf{P}<0.001 .{ }^{* *} \mathbf{P}<0.005\right)$ Trps1 KO (right) mice showed significantly reduced diameter of hair follicles compared with those of WT mice $(P=0.001)$. (C) Comparative analysis of diameter of hair shafts $(n=3$ per group, error bars represent $\mathrm{SD} ;{ }^{*} \mathrm{P}<0.001$. $\left.{ }^{*} \mathrm{P}<0.005\right)$ Trps1 KO (right) mice showed significantly reduced diameters of hair shaft compared with those of WT (left) mice $(P=0.005)$. (D) Comparative analysis of density of hair follicles $(n=3$ per group, error bars represent SD; ${ }^{*} \mathbf{P}<0.001$. ${ }^{* *} \mathbf{P}<0.005$ ) Density of hair follicles in WT (left) and Trps1 KO mice were not significantly different. some signaling pathways are affected. ${ }^{15,16}$ These findings demonstrate that the processes required for hair follicle growth are complicated. Wnt $/ \beta$-catenin signalling is essential for the initiation and maintenance of hair morphogenesis. ${ }^{17}$ In promoting dermal papilla cells properties to maintain $\mathrm{HF}$ regeneration, Wnt signalling through the $\beta$ catenin pathway played an important role. ${ }^{18}$ Trps1 may maintain mouse HFs through inhibition of canonical Wnt signalling and may operate as a major molecular regulator of $\mathrm{HF}$ regression. Remarkably, Wnt inhibitors, Wif1, Apcdd1 and Dkk4, were down-regulated in Trps1 KO mouse skin according to microarray hybridization analysis, suggesting that Trps 1 may repress the Wnt signalling pathway to develop normal hair growth. ${ }^{12}$ Therefore, we hypothesized that the miniaturization of hair follicles and hair shafts seen in Trps1 KO mice may be directly caused by inhibition of Wnt signalling. Nevertheless, to date, we have failed to detect any significant alteration in gene expression of Wnt inhibitors such as WifI, apcdd1 and Dkk4.

On the other hand, androgen (dihydrotestosterone: DHT) is believed to have an important role in transformation of scalp hair to vellus hair through dermal papilla morphogenesis. It has been reported that the dermal papilla from HF of a balding scalp contains higher levels of DHT than those from non-balding scalp. ${ }^{19}$ In addition, it has been shown that DHT disturbs the balance of Wnt agonist/antagonist in dermal papilla cells, down regulating Wnt10b mRNA. ${ }^{20}$

It is possible that Trps1 may act as a regulator of hair shaft formation. Trps1 functions in the normal hair cycle as a key molecule of $\mathrm{HF}$ regression by inhibiting Wnt signalling via androgen expression. This is in line with a previous report in which DHT abrogated the ability of DP cells to induce HF stem cells differentiation into a hair follicle lineage via inhibition of Wnt signaling. ${ }^{21}$

Although we could not show any significant change in androgen receptor expression in the transplanted skin from Trps1 KO mice compared with WT mice skin by immunofluorescence, it is possible that AR expression may be up regulated in Trps1 KO skin.

We have reported that Trps1 plays an important role in the morphogenesis of secondary but not primary HFs via an interaction with the BMP inhibitor Noggin. However, there was no significant difference in the density of hair follicles in the transplanted skin. To date, although we cannot explain the discrepancy clearly, it is possible that the interaction of the transplanted skin with normal dermal tissue in 
nude mice might have increased the hair follicle density. Injury to the recipient mouse skin could cause an upregulation in Wnt, resulting in signaling that promotes hair growth in the KO skin. Another possibility would be a mechanism by which Ambras syndrome is caused. Koa mice, a mouse model of AS, display hypertrichosis, which is an opposite phenotype of Trps1 KO mice although Trps1 expression levels are reduced. ${ }^{22}$ This molecular mechanism might explain the discrepancy between Trps1 KO and the transplanted skin. To further study the mechanism of how Trps1 regulates hair follicle growth after birth, we ought to investigate follicle growth using Trps 1 conditional KO mice where Trps 1 is specifically deficient in the skin.

In conclusion, soft hairs grew out of the transplanted skin from Trps1-deficient newborn mice. These hairs were thin, but were otherwise comparable with normal hairs, suggesting that Trps1 is unnecessary for hair growth itself, perhaps regulating only the diameter of hair shaft. Further study should be undertaken to elucidate the precise function of Trps 1 on hair development.

\section{References}

1. Canún S, Guevara-Sanginés EG, ElviraMorales A, et al. Hypertrichosisterminalis, gingival hyperplasia, and a characteristic face: a new distinct entity. Am J Med Genet A 2003;116:278-83.

2. Gai Z, Gui T, Muragaki Y. The function of TRPS1 in the development and differentiation of bone, kidney, and hair follicles. Histol Histopathol 2011;26: 915-21.

3. Merjaneh L, Parks JS, Muir AB, Fadoju D. A novel TRPS1 gene mutation causing trichorhinophalangeal syndrome with growth hormone responsive short stature: a case report and review of the literature. Int J Pediatr Endocrinol 2014;16.

4. Trippella G, Lionetti P, Naldini S, et al.
An early diagnosis of trichorhinophalangeal syndrome type 1: a case report and a review of literature. Ital J Pediatr 2018;44:138.

5. Vaccaro M, Tchernev G, Wollina U, et al. Trichorhinophalangeal syndrome. Open Access Maced J Med Sci 2017;5:486-9.

6. Kim YJ, Yoon B, Han K, Park BC. Comprehensive transcriptome profiling of balding and non-balding scalps in trichorhinophalangeal syndrome type I patient. Ann Dermatol 2017;29:597601.

7. Lüdecke HJ, Schaper J, Meinecke P, et al. Genotypic and phenotypic spectrumin tricho-rhino-phalangeal syndrome types I and III. Am J Hum Genet 2001;68:81-91.

8. Malik TH, Von Stechow D, Bronson RT, et al. Deletion of the GATA domain of TRPS1 causes an absence of facial hair and provides new insights into the bone disorder in inherited tricho-rhinophalangeal syndromes. Mol Cell Biol 2002;22:8592-600.

9. Wen TC, Li YS, Rajamani K, et al. Effect of Cinnamomum osmophloeum Kanehira leaf aqueous extract on dermal papilla cell proliferation and hair growth. Cell Transplant 2018;27:25663.

10. Chen D, Jarrell A, Guo C, et al. Dermal $\beta$-catenin activity in response to epidermal Wnt ligands is required for fibroblast proliferation and hair follicle initiation. Development 2012;139:1522-33.

11. Sun Y, Nakanishi M, Sato F, et al. Trps 1 deficiency inhibits the morphogenesis of secondary hair follicles via decreased Noggin expression. Biochem Biophys Res Commun 2015;456:721-6.

12. Fantauzzo KA, Christiano AM. Trps1 activates a network of secreted Wnt inhibitors and transcription factors crucial tovibrissa follicle morphogenesis. Development 2012;139:203-14.

13. Suemoto H, Muragaki Y, Nishioka K, et al. Trps1 regulates proliferation and apoptosis of chondrocytes through
Stat3 signaling. Dev Biol 2007;312: 572-81.

14. Zhu K, Xu C, Liu M, et al. Hairless controls hair fate decision via $\mathrm{Wnt} / \beta$ catenin signaling. Biochem Biophys Res Commun 2017;491:567-70.

15. Botchkarev VA, Kishimoto J. Molecular control of epithelial-mesenchymal interactions during hair follicle cycling. J Investig Dermatol Symp Proc 2003;8:46-55.

16. Roh C, Tao Q, Lyle S. Dermal papillainduced hair differentiation of adult epithelial stem cells from human skin. Physiol Genom 2004;19:207-17.

17. Millar SE. Molecular mechanisms regulating hair follicle development. J Invest Dermatol 2002;118:216-25.

18. Xiong Y, Liu Y, Song Z, et al. Identification of $\mathrm{Wnt} / \beta$-catenin signaling pathway in dermal papilla cells of human scalp hair follicles: TCF4 regulates the proliferation and secretory activity of dermal papilla cell. J Dermatol 2014;41:84-91.

19. Lai JJ, Chang P, Lai KP, et al. The role of androgen and androgen receptor in skin-related disorders. Arch Dermatol Res 2012;304:499-510.

20. Leirós GJ, Ceruti JM, Castellanos ML, et al. Androgens modify Wnt agonists/antagonists expression balance in dermal papilla cells preventing hair follicle stem cell differentiation in androgenetic alopecia. Mol Cell Endocrinol 2017;439:26-34.

21. $\mathrm{Xu} \mathrm{Z}$, Wang $\mathrm{W}$, Jiang $\mathrm{K}$, et al. Embryonic attenuated $\mathrm{Wnt} / \beta$-catenin signaling defines niche location and long-term stem cell fate in hair follicle. Elife 2015;4:e10567.

22. Fantauzzo KA, Tadin-Strapps M, You $\mathrm{Y}$, et al. A position effect on TRPS1 is associated with Ambras syndrome in humans and the Koala phenotype in mice. Hum Mol Genet 2008;17:353951. 